\title{
Loss of LDAH associated with prostate cancer and hearing loss
}

Benjamin B. Currall ${ }^{1,2, \dagger}$, Ming Chen ${ }^{2,3}$, Richard C. Sallari ${ }^{4,5}$, Maura Cotter ${ }^{6}$, Kristen E. Wong ${ }^{1}$, Nahid G. Robertson ${ }^{1}$, Kathryn L. Penney ${ }^{2,7,8}$, Andrea Lunardi ${ }^{2,3}$, Markus Reschke ${ }^{2,3}$, Ann E. Hickox ${ }^{2,9,10}$, Yanbo Yin ${ }^{10,11}$, Garrett T. Wong ${ }^{12}$, Jacqueline Fung ${ }^{3}$, Kerry K. Brown ${ }^{13}$, Robin E. Williamson ${ }^{13}$, Nicholas A. Sinnott-Armstrong ${ }^{4}$, Tammy Kammin ${ }^{1}$, Andrew Ivanov ${ }^{1}$, Cinthya J. Zepeda-Mendoza ${ }^{1,2}$, Jun Shen 2,14,15,16 , Bradley J. Quade 2,14, Sabina Signoretti ${ }^{2,14,17}$, Kathleen S. Arnos ${ }^{18}$, Alexander S. Banks ${ }^{2,19}$, Nikolaos Patsopoulos 2,5,12,20, M. Charles Liberman'2,9,10,11, Manolis Kellis ${ }^{4,5}$, Pier Paolo Pandolfi ${ }^{2,3}$ and Cynthia C. Morton ${ }^{1,2,5,9,14,16,21, *}$

\footnotetext{
${ }^{1}$ Department of Obstetrics, Gynecology and Reproductive Biology, Brigham and Women's Hospital, Boston, MA 02115, USA, ${ }^{2}$ Harvard Medical School, Boston, MA 02115, USA, ${ }^{3}$ Cancer Research Institute, Department of Medicine and Pathology, Beth Israel Deaconess Medical Center, Boston, MA 02215, USA, ${ }^{4}$ Computer Science and Artificial Intelligence Laboratory, Massachusetts Institute of Technology, Cambridge, MA 02142, USA, ${ }^{5}$ Program in Medical and Population Genetics, Broad Institute of MIT and Harvard, Cambridge, MA 02142, USA, ${ }^{6}$ Center for Molecular Oncologic Pathology, Dana Farber Cancer Institute, Boston, MA 02215, USA, ${ }^{7}$ Channing Division of Network Medicine, Department of Medicine, Brigham and Women's Hospital, Boston, MA 02115, USA, ${ }^{8}$ Department of Epidemiology, Harvard T.H. Chan School of Public Health, Boston, MA 02115, USA, ${ }^{9}$ Program in Speech and Hearing Bioscience and Technology, Harvard-MIT Division of Health Sciences and Technology, Cambridge, MA 02138, USA, ${ }^{10}$ Eaton-Peabody Laboratory, Massachusetts Eye and Ear Infirmary, Boston, MA 02114, USA, ${ }^{11}$ Department of Otology and Laryngology, Harvard Medical School, Boston, MA 02115, USA, ${ }^{12}$ Department of Neurology, Brigham and Women's Hospital, Harvard Medical School, Boston, MA 02115, USA, ${ }^{13}$ Department of Genetics, Harvard Medical School, Boston, MA 02115, USA, ${ }^{14}$ Department of Pathology, Brigham and Women's Hospital, Boston, MA 02115, USA, ${ }^{15}$ Laboratory for Molecular Medicine, Partners HealthCare Personalized Medicine, Cambridge, MA 02139, USA, ${ }^{16}$ Harvard Medical School Center for Hereditary Deafness, Boston, MA 02115, USA, ${ }^{17}$ Department of Medical Oncology, Dana-Farber Cancer Institute, Boston, MA 02215, USA,
}

${ }^{\dagger}$ Benjamin B. Currall, http://orcid.org/0000-0001-6023-6400

Received: July 18, 2018. Revised: August 27, 2018. Accepted: August 29, 2018

@ The Author(s) 2018. Published by Oxford University Press. All rights reserved.

For Permissions, please email: journals.permissions@oup.com 
${ }^{18}$ Department of Science, Technology, \& Mathematics, Gallaudet University, Washington, DC 20002, USA, ${ }^{19}$ Division of Endocrinology, Diabetes and Hypertension, Department of Medicine, Brigham and Women's Hospital, Boston MA 02115, USA, ${ }^{20}$ Division of Genetics, Department of Medicine, Brigham and Women's Hospital, Boston, MA 02115, USA and ${ }^{21}$ Division of Evolution and Genomic Sciences, Manchester Academic Health Science Centre, University of Manchester, Manchester M13 9NT, UK

*To whom correspondence should be addressed at: Brigham and Women's Hospital, 77 Avenue Louis Pasteur, Boston, MA 02115, USA. Tel: 617-525-4535; Fax: 617-525-4533; Email: cmorton@bwh.harvard.edu

\begin{abstract}
Great strides in gene discovery have been made using a multitude of methods to associate phenotypes with genetic variants, but there still remains a substantial gap between observed symptoms and identified genetic defects. Herein, we use the convergence of various genetic and genomic techniques to investigate the underpinnings of a constellation of phenotypes that include prostate cancer (PCa) and sensorineural hearing loss (SNHL) in a human subject. Through interrogation of the subject's de novo, germline, balanced chromosomal translocation, we first identify a correlation between his disorders and a poorly annotated gene known as lipid droplet associated hydrolase (LDAH). Using data repositories of both germline and somatic variants, we identify convergent genomic evidence that substantiates a correlation between loss of LDAH and PCa. This correlation is validated through both in vitro and in vivo models that show loss of LDAH results in increased risk of PCa and, to a lesser extent, SNHL. By leveraging convergent evidence in emerging genomic data, we hypothesize that loss of LDAH is involved in PCa and other phenotypes observed in support of a genotype-phenotype association in an n-of-one human subject.
\end{abstract}

\section{Introduction}

Decreasing costs of genomic sequencing have led to a rapidly expanding catalog of both pathogenic and benign variants associated with human traits and disorders, making it possible to envision genome-informed personalized medicine. Nonetheless, determining pathogenicity of variants in individuals remains a daunting challenge. Rare variants are often attractive targets because they may be analyzed under a monogenic model. However, small sample size, incomplete penetrance and background biases make it difficult to impute the impact of these novel variants to the general population (1). On the opposite end of the spectrum, genome-wide association studies (GWASs) have established the correlation between hundreds of common variants and common diseases; however, these common variants tag loci with diminishingly small and uncertain effects and only explain a fraction of human heritability (2-4). However, the genetic impact of $n$-of-one studies has been increasingly elucidated through leveraging the mutual intersection of monoand poly-genetic studies (4-6).

In this study, we employed a convergent genomic approach to identify an association between reduced expression of a poorly annotated gene, known as lipid droplet associated hydrolase (LDAH), and a syndromic phenotype in a single individual. Through the Developmental Genome Anatomy Project (DGAP, www.dgap.harvard.edu), we first identified a putative association of LDAH with prostate cancer (PCa) and sensorineural hearing loss (SNHL) in a human subject (DGAP056) with a de novo germline balanced chromosomal abnormality (BCA). We then amassed convergent genomic evidence showing population level associations between LDAH expression and occurrence of PCa. We further identified loss of LDAH in both tissues and cell lines derived from human PCa, and generated a $\mathrm{Ldah}^{-/}$mouse model, which recapitulated many of the clinical findings in DGAP056. This investigation elucidates a causal correlation between reduced expression of LDAH and PCa in in vivo and in vitro studies in both humans and mice.

\section{Results}

Association of loss of LDAH expression due to a de novo variant with early onset $\mathrm{PCa}$

DGAP056 first presented with a constellation of congenital abnormalities including profound SNHL, craniofacial dysmorphism, hypospadias and mitral valve prolapse. During the course of this study, DGAP056 was diagnosed with PCa at age 38 years, over two standard deviations below the mean age-of-diagnosis at 70 years (Fig. 1 and Supplementary Material, Fig. S1), resulting subsequently in a radical prostatectomy (see detailed history in Supplementary Information). Histological sections from DGAP056's formalin-fixed paraffin-embedded prostate tissue block were haematoxylin and eosin (H\&E) stained to visualize abnormal structure (Fig. 1A). Hyperplasia within several glands containing prominent nucleoli was observed in concert with the histopathology report. Given the multiple congenital anomalies and early-onset $\mathrm{PCa}$, we explored the possibility of a genetic germline origin of the phenotype in DGAP056.

Upon enrollment at 21 years of age, DGAP056's karyotype was interpreted as 46,XY,t(2;13)(p24;q21)dn, indicating an apparently balanced de novo chromosome translocation. Chromosomal breakpoints were mapped using fluorescence in situ hybridization (FISH) with a series of probes of labeled bacterial artificial chromosome (BAC) clones. FISH with BAC RP11-31005, which localizes to 2p24.1, hybridized to the normal chromosome 2, the $\operatorname{der}(2)$ and the $\operatorname{der}(13)$ (Fig. S2). Likewise, BAC RP11-19D22, which localizes to 13q22.2-q22.3, hybridized to the normal chromosome 13, the der(13) and the der(2) (Data not shown). These split BACs refined the translocation breakpoints to $2 \mathrm{p} 24.1$ and 13q22.2-q22.3. Sanger sequencing further resolved the 


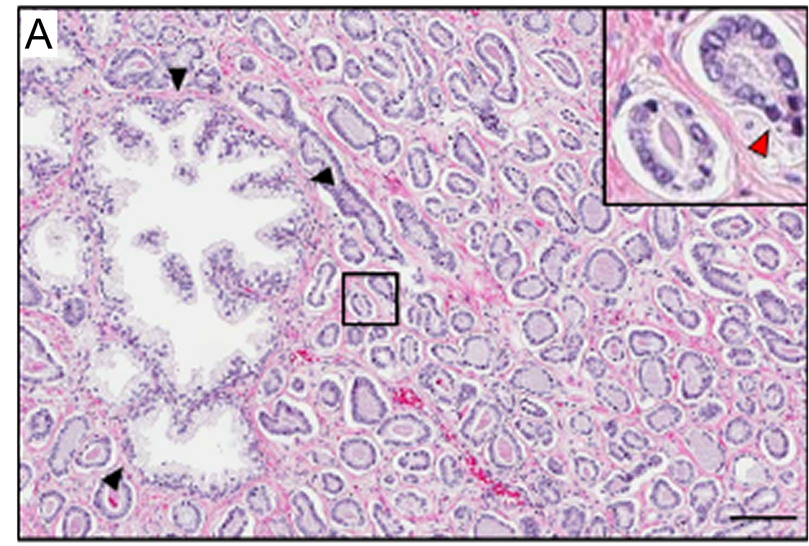

$\mathrm{B}$
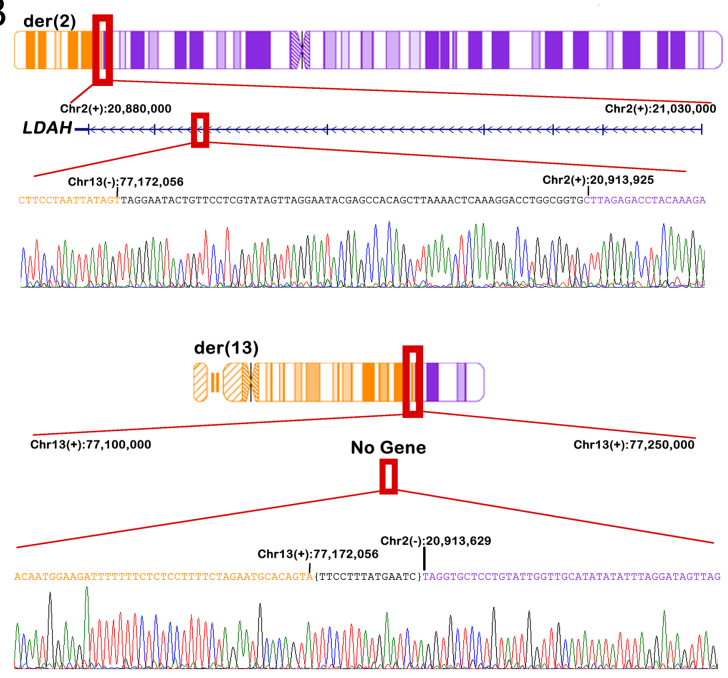

Figure 1. DGAP056 PCa and breakpoint sequencing. (A) H\&E stain of section from DGAP056 prostate obtained during radical prostatectomy, indicating benign prostate gland (black arrowheads) compared to adenocarcinoma with many neoplastic glands containing prominent nucleoli on the right (insert, red arrowheads) (scale bar indicates $100 \mu \mathrm{m}$ ). (B) Ideograms of $\operatorname{der}(2)$ (top) and $\operatorname{der}(13)$ (bottom) chromosomes with Sanger sequencing chromatographs of breakpoints disrupting LDAH (HGNC:26145, previously designated as FLJ21820 and C2orf43). Segments containing chromosomes 2 (purple) or 13 (orange) sequence are indicated. Red boxes indicate positions shown in higher resolution at the genic and nucleotide levels. Nucleotides of breakpoints are noted along with a $68 \mathrm{bp}$ insertion of nontemplated sequence (nucleotides in black lettering) in the der(2) and microhomology (nucleotides delineated with curly brackets) in the der(13).

breakpoints to nucleotide level and, using Next-Generation Cytogenetic Nomenclature (7), the karyotype is defined as follows: 46,XY,t(2;13)(p24.1;q22.2)dn.seq[GRCh37/hg19] $\mathrm{t}(2 ; 13)(13 q$ ter$>13 q 22.2(77,172,056)::$ TAA...GTG $\{68\}:: 2 p 24.1(20,913,647)->2$ qter; 13pter->13q22.2(77,172,04\{5-9\})::2p24.1(20,913,6\{43-39\})->2pter) dn (Fig. 1B).

This karyotype indicates that no annotated genes were disrupted in the region of the chromosome 13 breakpoint; however, the chromosome 2 breakpoint disrupts the poorly annotated gene encoding LDAH.

To determine the functional effect of the chromosome translocation, LDAH transcription was evaluated in an EpsteinBarr virus-immortalized B-lymphoblastoid cell line (LCL) derived from DGAP056's whole blood. Rapid amplification of cDNA ends (RACE) reveled both the normal full-length LDAH transcript (ENST00000237822.7) as well as two novel abnormal transcripts
(Supplementary Material, Fig. S3A). Quantitative analysis of these transcripts showed reduced expression of the full-length LDAH transcript (Supplementary Material, Fig. S3B). Western blot analysis of protein extracted from DGAP056's LCL showed a reduction in normal LDAH protein, but no abnormal protein products were discernable (Supplementary Material, Fig. S3C). The reduced expression at both RNA and protein levels suggests a haploinsufficiency effect from disruption of a single coding LDAH allele in the etiology of DGAP056's phenotype including the early-onset PCa.

\section{Association of a common germline variant in $\mathrm{PCa}$ populations with loss of $\mathrm{LDAH}$}

To investigate a relationship between LDAH disruption and PCa, we interrogated population level association studies between germline genomic variation and abnormal phenotypes using public databases. Multiple disease associating genetic databases, including several GWASs, were integrated into browser extensible data and visualized in the University of California Santa Cruz Genome Browser using the human GRCh37/hg19 reference file in the linkage-disequilibrium (LD) region of European ancestry for LDAH (Chr2:20,849,778-21,052,988; Supplementary Material, Fig. S4) $(8,9)$. The LD block for LDAH was notable for a variety of reported traits (e.g. low-density lipoprotein, cholesterol levels, triglyceride levels, lymphocyte counts, digit-length ratio and brachial arm circumference). Nominal genome-wide significance for PCa was identified for SNP rs13385191 G, which is located between exons 6 and 7 of the full-length LDAH transcript (NM_021925.3, Fig. 2A and Supplementary Material, Fig. S4) (10). A systematic analysis of mapped genetic association studies and the literature revealed six other GWASs or candidate association studies in European, Chinese and African populations that examined association of this locus with $\mathrm{PCa}$ (11-16). Of these seven studies, five examined the association between the rs13385191 locus and a PCa diagnosis, while two examined associations with more aggressive forms of PCa. A retrospective meta-analysis was performed stratifying these studies on a diagnosis of PCa and aggressive PCa. While the aggressive $\mathrm{PCa}$ studies did not meet genome-wide significance of disease association, genome-wide significance $(P=2.1 \mathrm{e}-08)$ was observed between the risk allele (rs13385191 G) and the diagnosis of PCa with a combined increased odds ratio (OR) of 1.17 [95\% confidence interval (CI), 1.12-1.23; Fig. 2A]. The metaanalysis reported herein confirms previous studies showing genome-wide significance in the association of $L D A H$ variants with PCa.

To determine the risk allele's association with changes in expression levels of LDAH, the expression quantitative trait locus (eQTL) of rs1338191 was assessed in normal prostate tissues ( $\mathrm{n}=87$ ) in the Genotype-Tissues Expression (GTEx) database. These data show that subjects carrying both copies of the PCa risk allele (rs13385191 G) had significant specific downregulation of LDAH $(P=5.0 \mathrm{e}-5)$ in their prostates compared to subjects homozygous for the reference allele (rs13385191 A) (Fig. 2B). The combined GWAS and eQTL data corroborated the findings in DGAP056, substantiating that both measures of population-level variants associated with downregulation of LDAH correlate with an increased risk of PCa. Similar to the majority of GWAS alleles, the rs13385191 $\mathrm{G}$ risk allele has a small, but significant increase in $\mathrm{PCa}$ risk $(\mathrm{OR}=1.17)$. 


\begin{tabular}{lccccccc}
\hline \multicolumn{7}{c}{ Meta-Analysis of Prostate Cancer GWAS and Replication Studies Acr oss Ethnicities } \\
\hline \multicolumn{1}{c}{ Population } & PMID & $\begin{array}{c}\text { Study } \\
\text { Design }\end{array}$ & $\begin{array}{c}\text { Best } \\
\text { Marker }\end{array}$ & $\begin{array}{c}\text { Risk } \\
\text { Allele }\end{array}$ & OR & Weight & P-value \\
Japanese & 20676098 & GWAS & rs 13385191 & G & 1.15 & 2.1 & $7.5 \mathrm{e}-08$ \\
Chinese & 22662242 & Candidate & rs 13385191 & G & 1.33 & 0.6 & $8.6 \mathrm{e}-05^{\dagger}$ \\
African-American & 21637779 & Candidate & rs 340623 & T & 1.15 & 1.1 & $3.8 \mathrm{e}-03^{\dagger}$ \\
European & 22056501 & Candidate & rs 13385191 & G & 1.15 & 0.9 & $1.1 \mathrm{e}-02^{\dagger}$ \\
Northern Chinese & 23803082 & Candidate & rs 13385191 & G & 1.12 & 0.3 & $3.4 \mathrm{e}-01$ \\
\hline
\end{tabular}
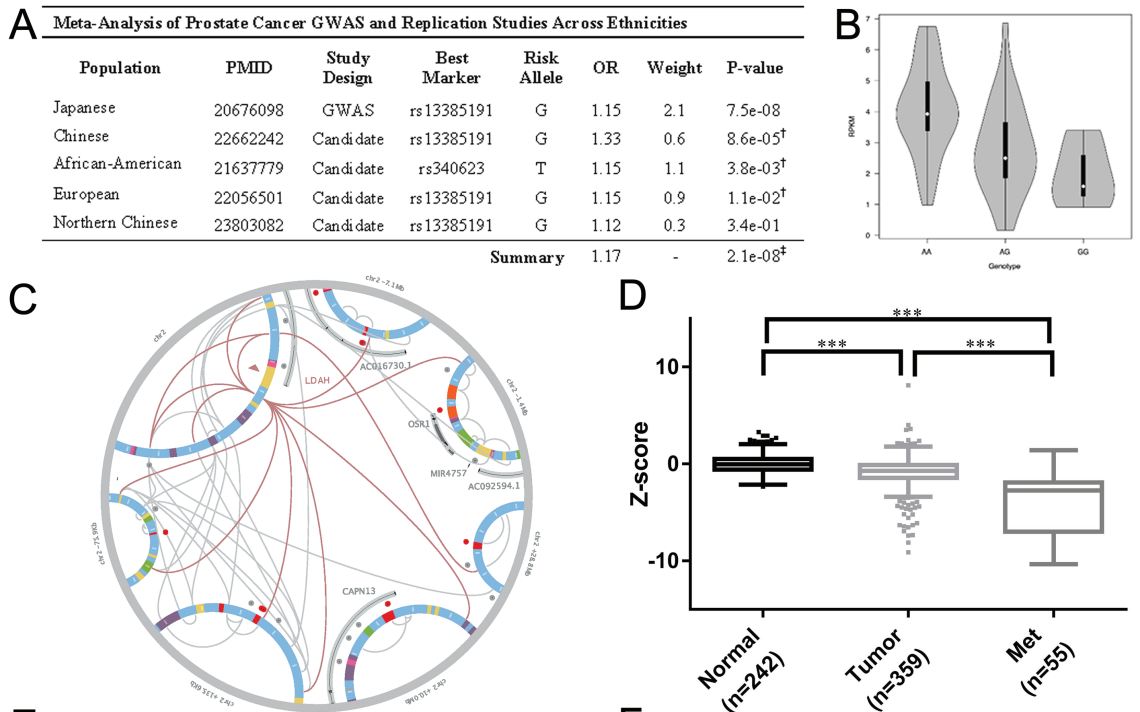

$\mathrm{E}$

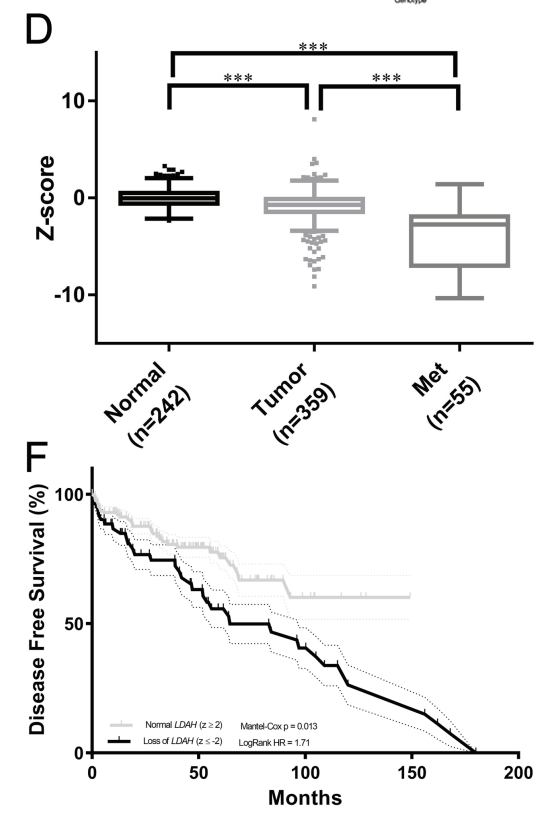

Figure 2. GWAS and somatic mutation association of LDAH with PCa. (A) Meta-analysis of GWAS and candidate association studies ( ${ }^{\ddagger}$ genome-wide, ${ }^{\dagger}$ candidate-level significance). (B) eQTL violin plot of LDAH expression in subjects with either reference (rs13385191 A) and/or risk (rs13385191 G) alleles. (C) Plexus diagram showing LDAH locus with direct (pink Bezier curve) and indirect (grey Bezier curve) chromatin loops. Putative driver (red dots) and passenger mutations (grey dots) with chromatin states in the LDAH plexus including promoter-like histone modifications (red), enhancer (yellow), dyadic (orange), transcribed (green), repressed (purple) and poised (pink) regions. (D) Relative expression of LDAH in benign (black), primary (grey) and metastatic (light grey) prostate tissue (Tukey range with outliers indicated). (E) Percent of subjects who have significant dysregulation of a given gene within their prostate tumor. Relative rank order (arrows) and percent of subjects with dysregulation of LDAH and established prostate oncogene (ERG) and tumor suppressor (PTEN, NKX3-1) genes along with top and bottom $1^{\text {st }}$ (dotted lines) and $5^{\text {th }}$ (dashed lines) percentiles. (F) Disease-free survival of subjects with normal compared to significantly reduced $(\mathrm{z} \leq-2)$ LDAH expression. Significance indicated by ${ }^{*} \mathrm{P} \leq 0.05$, ${ }^{* *} \mathrm{P} \leq 0.01$ and $* * P \leq 0.001$.

\section{Association of somatic mutations in PCa with loss of LDAH expression}

We next investigated whether the relationship between LDAH and $\mathrm{PCa}$ in germline tissues extends to somatic mutations in prostate tumors. Initial analysis from The Cancer Genome Atlas showed no significant increase of prostate-specific somatic mutations or copy number alterations in LDAH exons. This analysis was then extended to the entire regulatory region of LDAH using the plexus reoccurrence test (PRT) (17). Using whole genome sequencing data from adenocarcinomas compared to adjacent benign prostate tissue $(\mathrm{n}=55)$, a 10 -fold significant enrichment $(P=0.004)$ of somatic mutations in LDAH regulatory regions was observed in adenocarcinoma tissue (Fig. 2C). This significant increase in somatic mutations in regulatory regions suggests that dysregulation of LDAH in prostate tumors is common.

To determine if these genomic changes are related to a functional effect, we examined changes in LDAH levels in prostate tumor tissues. Starting with expression data deposited in the Gene Expression Omnibus (GEO) from four of the largest microarray analysis studies of PCa, LDAH differential expression was analyzed from both benign and tumor samples (18-21). All studies consistently show significant down-regulation of LDAH in primary tumors, with a combined $\mathrm{z}$-score mean $=-0.84$
$(P=6.1 \mathrm{e}-14)$ (Fig. 2D and Supplementary Material, Fig. S5). LDAH down-regulation was even more dramatic in metastatic tumors, which had significantly less expression than either benign (combined $z$-score mean $=-3.9 ; P=4.2 \mathrm{e}-43$ ) or primary tumor tissues $(P=2.0 \mathrm{e}-30)$. Cross comparison across the entire shared gene set showed that LDAH was one of the most frequently down-regulated genes $(z$-score $\leq-2.0)$ in both primary $(9.5 \%$ of tumors, $96^{\text {th }}$ percentile of all genes) and metastatic $\left(69 \%, 99^{\text {th }}\right.$ percentile) prostate tissues (Fig. 2E and Supplementary Material, Fig. S5). Notably, LDAH was more frequently down-regulated in both primary and metastatic tumors $\left(15 \%, 97^{\text {th }}\right.$ percentile) than some well-established PCa tumor suppressors including PTEN $\left(13 \%, 96^{\text {th }}\right.$ percentile) and NKX3-1 $\left(4.63 \%, 84^{\text {th }}\right.$ percentile). These data further substantiate that significant transcriptional downregulation of LDAH is a common event in both primary and metastatic prostate tumors.

We also investigated the relationship of LDAH expression with PCa morbidity (Fig. 2F). Using the aforementioned PCa microarray data, disease-free survival times in subjects with significant down-regulation $(z \leq-2)$ of LDAH was compared to those with normal or elevated LDAH expression. This analysis revealed a significantly shortened interval (hazard ratio $=1.71$; $P=0.013)$ in cancer-free survival in subjects with reduced LDAH expression in their prostate tumors, suggesting that not only is LDAH reduction associated with the presence of $\mathrm{PCa}$, 

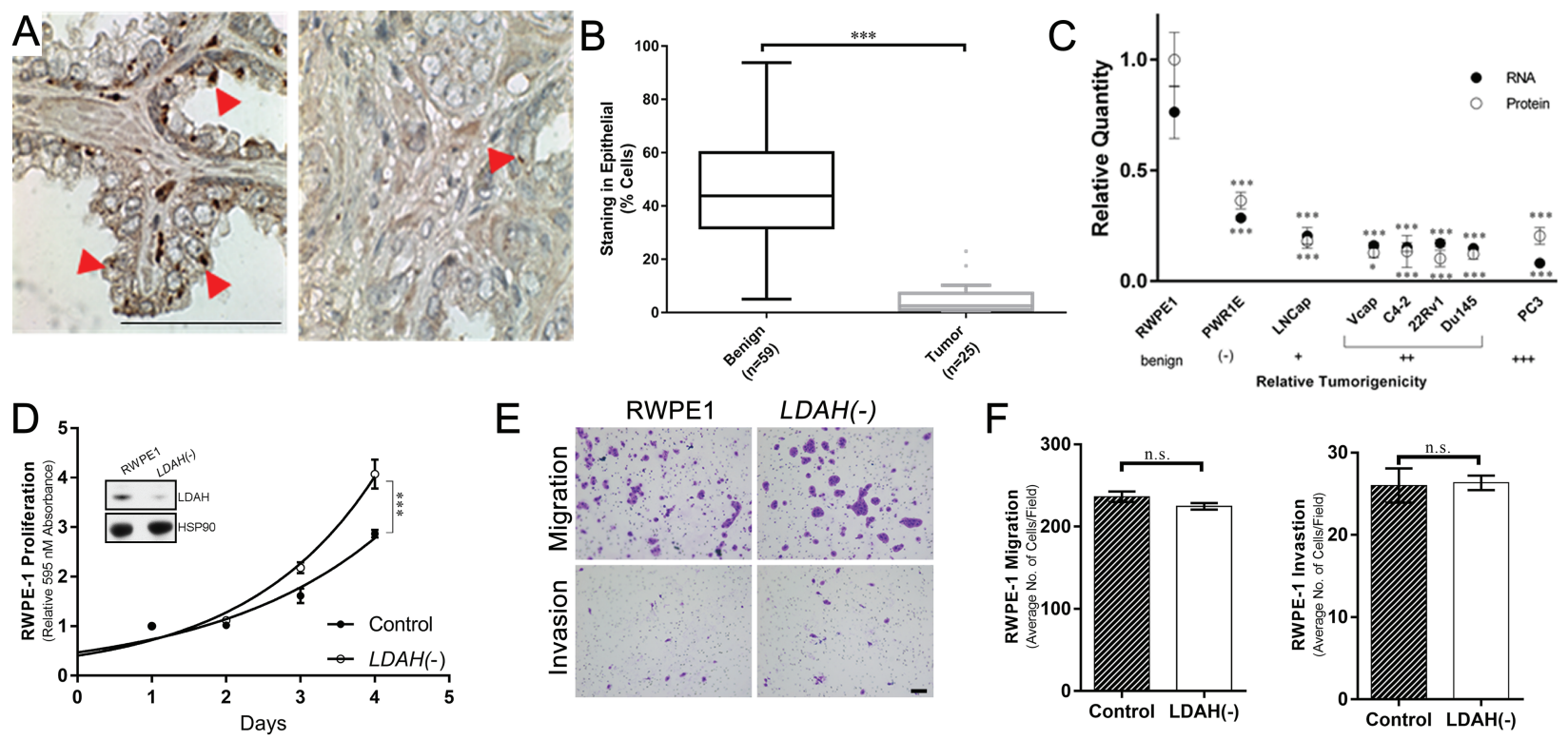

E
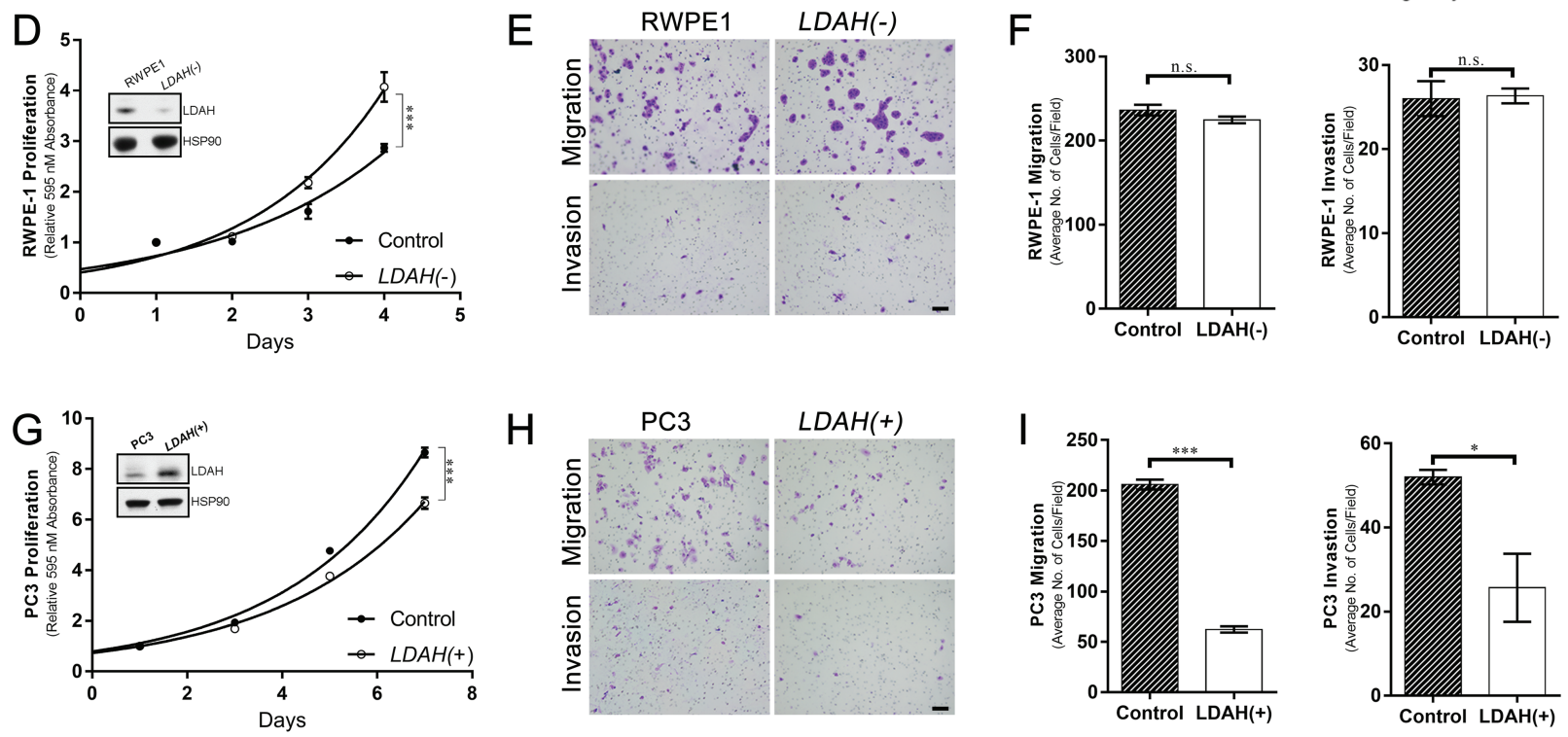

Figure 3. Expression of LDAH in prostate tissues. (A) IHC of benign (left) and tumor (right) prostate tissue microarray detected with anti-LDAH antibody, showing punctate cytoplasmic staining of epithelial cells (red arrowhead). (B) Quantitative analysis of percent of epithelial cells containing punctate LDAH staining in benign (black) and tumor prostate (grey). (C) Quantitative expression of RNA (filled circles) and protein (open circles) of LDAH from prostate cell lines, grouped by relative tumorigenicity indicated as benign, (-) for hyperplastic and,,++++++ for increasing levels of tumorigenicity (see Supplementary Material, Table S1) with significance compared to the normal-like RWPE1 cell line. (D-I) Proliferation, migration and invasion assays after in vitro manipulation of LDAH expression. Knockdown of LDAH (LDAH(-), D-F) was performed in the RWPE1 cell line, confirmed with western blot (D inset), then measured for proliferation (D) as well as microscopic (E) and quantitative (F) analysis of migration and invasion. Similarly, stable transfection of LDAH (LDAH(+), G-I) was performed in the tumorigenic PC3 cell line, confirmed with western blot ( $G$ inset), then measured for proliferation $(\mathrm{G})$, microscopic $(\mathrm{H})$ and quantitative (I) analysis of migration and invasion. Significance indicated by ${ }^{*} \mathrm{P} \leq 0.05$, ${ }^{* *} \mathrm{P} \leq 0.01$ and *** $P \leq 0.001$, inset black bar indicates $100 \mu \mathrm{m}$.

but may also have an association with severity of disease. Given convergent genomic evidence observed in rare germline, common germline and recurrent somatic variants, it appears highly likely that loss of LDAH is pathogenic in PCa.

\section{LDAH levels are reduced in prostate tumor tissues}

For a more direct examination of LDAH in neoplasia, we examined changes to LDAH protein levels in prostate tissue cores from histologic sections of both benign and tumor tissues (Fig. 3A and B). In benign cores, a high percentage of luminal epithelial cells show positive punctate cytoplasm with LDAH immunostaining (46.5\%), which was not observed in surrounding stroma. In tumor cores, a significant reduction in the percentage of epithelial cells shows this positive immunostaining $(4.57 \%, P=1.17 \mathrm{e}-22)$. Consistent with the transcriptional changes observed in the microarrays, histologic staining suggests loss of LDAH is a frequent occurrence in prostate tissue and further localizes this loss to luminal epithelial cells, which are the cells most likely to give rise to prostate adenocarcinoma (22). Akin to our genomic analysis, these studies indicate that prostate tumors have a reduced level of LDAH.
Similar to prostate histological studies, we analyzed LDAH expression levels in cell lines derived from both benign and neoplastic prostate tissues. We extracted both RNA and protein from eight independent prostate-derived cell lines with various oncogenic properties (Fig. 3C and Supplementary Material, Table S1). PC3 cells, the most tumorigenic prostate cell line, had significantly lower $(P<0.05)$ LDAH transcript and protein levels when compared to the non-tumorigenic prostate cell line RWPE1. This reduction of LDAH expression and protein correlates with increased tumorigenicity of the cell lines with a marked reduction in every tumorigenic cell line compared to RWPE1 cells. In contrast, RWPE1 cells show high levels of LDAH protein and transcript levels. This endogenous reduction of LDAH expression suggests that loss of LDAH is a characteristic of the biology in prostate tumors.

\section{LDAH suppression increases tumorigenicity in prostate cell lines}

To determine whether loss of LDAH expression has a direct effect on tumorigenicity, we analyzed several prostate cell lines taking advantage of their differential LDAH expression (Fig. 3D-I). For RWPE1 cells, a non-tumorigenic cell line with a high level 
of expression of $L D A H$, transient silencing of LDAH transcripts, using shRNA, resulted in significant increased proliferation (1.5fold, $P<0.001$ ) within 4 days of transfection. Interestingly, this increase in proliferation were not accompanied by any change in migration or invasion. We then used PC3 cells, a highly tumorigenic cell line with a low level of LDAH expression, to overexpress LDAH using a stable transfection vector. Overexpression of LDAH resulted in significantly decreased proliferation $(P<0.001)$ within 7 days of transfection. Furthermore, overexpression of LDAH in PC3 cells significantly reduced migration and invasion $(P<0.05$ in all studies) effectively reducing some of the tumorigenic properties of PC3 cells. These in vitro data further implicate a causal role of $L D A H$ in $\mathrm{PCa}$, confirming the association between reduced LDAH expression and PCa observed in DGAP056, association studies and somatic mutations in prostate tumors.

\section{Ldah $^{\text {- }}$ mice develop PCa, SNHL and higher body mass}

To determine if PCa and other pleiotropic findings in DGAP056 replicate with in vivo loss of $L D A H$, we generated a mouse model with constitutional knockout of Ldah. The knockout mouse was developed through insertion of a reporter cassette within the coding frame of the mouse LDAH ortholog, 1110057K04Rik (MGI:1916082, referred to herein as $\mathrm{Ldah}^{-/}$, Supplementary Material, Fig. S6) (23). Analysis of various tissues demonstrated that $\mathrm{Ldah}^{-1-}$ mice had negligible levels of Ldah transcript or protein expression, confirming successful knockout of Ldah in this mouse line (Supplementary Material, Fig. S6).

$\mathrm{Ldah}^{-/}$phenotyping was guided by the clinical symptoms observed in DGAP056. There were no apparent correlative gross congenital phenotypic abnormalities in $\mathrm{Ldah}^{-1-}$ mice. There was also no skew in the normal Mendelian inheritance ratio of the Ldah allele in either males or females. Systematic measurement of body weight over time revealed that by 8 months of age, female $\mathrm{Ldah}^{-1}$ mice (but not males) were significantly heavier $(P<0.0001)$ than their $\mathrm{Ldah}^{+/+}$littermates (Supplementary Material, Fig. S7A). Auditory function was also assessed and a significant 10-20 dB elevation in hearing threshold in 1-yearold male $\mathrm{Ldah}^{-1}$ mice $(\mathrm{P}<0.01)$ was observed when compared to their $\mathrm{Ldah}^{+/+}$littermates (Supplementary Material, Fig. S7B). Interestingly, while 1-year-old female $\mathrm{Ldah}^{-/}$mice did show a partial loss of hearing $[P<0.05$ in auditory brainstem responses (ABRs) only, predominately in high frequency tones] this was less severe than the hearing loss observed in males. While dysregulation of lipid metabolism (as observed in the female mice and genomic studies) and hearing loss (as observed in DGAP056 and male mice) seem to correlate with loss of $\mathrm{LDAH}$, a more dramatic phenotype was observed in prostate tumorigenicity.

The next step was a detailed histological examination of prostate tissues in our $\mathrm{Ldah}^{-/-}$mouse model. A genotype-blinded analysis was performed of H\&E- and Ki-67-stained histologic prostate sections of 12-month-old (mean age $=12.1 \pm 1.6$ months) mice (Fig. 4 and Supplementary Material, Fig. S8) (24-26). Prostate tissues were separated into anterior (AP), ventral (VP) and dorsolateral (DLP) lobes, all of which were scored as either benign, mouse prostate intraepithelial neoplasia (mPIN and further subgraded into mPIN-I to mPIN-IV), or invasive (subgraded into microinvasive or invasive). Ldah ${ }^{-1}$ had a significant $(\mathrm{OR}=3.23,95 \% \mathrm{CI}=1.12-9.34, \mathrm{P}=0.037)$ enrichment in combined mPIN and invasive lesions across all three lobes compared to their $\mathrm{Ldah}^{+/+}$littermates. Further, this enrichment was consistent across all three lobes, with $\mathrm{Ldah}^{-1}$ mice displaying more high-grade lesions (mPIN III-IV)

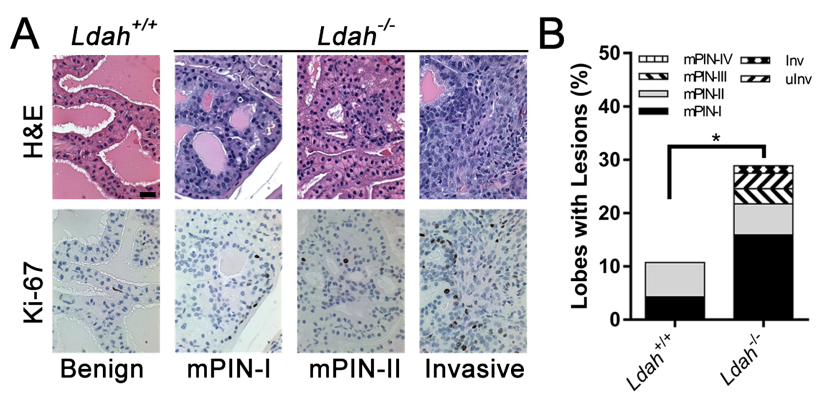

Figure 4. Prostate tumors in Ldah $/$ mice. (A) H\&E- (top) and Ki-67- (bottom) stained sections of mouse prostate tissue showing benign histology in $\mathrm{Ldah}^{+/+}$ mice (left) and in increasingly more aggressive tumors (middle and right). (B) Quantitative analysis of the percent of prostate lobes with at least one lesion from $\mathrm{Ldah}^{+/+}$and $\mathrm{Ldah}^{-/-}$mice. Significance indicated by ${ }^{*} \mathrm{P} \leq 0.05$, inset black bar indicates $100 \mu \mathrm{m}$.

in all three lobes of the prostate whereas lesions in $\mathrm{Ldah}^{+/+}$ were lower-grade (mPIN I-II) and restricted to the AP lobe. More impressively, $10 \%$ of $\mathrm{Ldah}^{-/}$mice had invasive prostate lesions, which were found exclusively in the $\mathrm{Ldah}^{-/}$dorsolateral lobe, which is the mouse lobe most similar biochemically to human prostate (Supplementary Material, Table S2) (27). The $\mathrm{Ldah}^{-/}$ mouse model showed that loss of Ldah expression significantly increases the probability of developing PCa.

\section{Discussion}

In this study, we show that loss of LDAH is linked to increased risk of PCa as well as SNHL. We first identified the association of LDAH with these symptoms in a gene discovery experiment by analyzing a de novo germline BCA in a human subject, DGAP056. Through resolving this chromosomal translocation to nucleotide level and performing expression assays, we were able to incriminate LDAH in DGAP056's disorder. However, given no other familial carriers of this BCA and the low probability of finding a similar BCA in the greater population, it was not possible to determine the statistical likelihood of this association. To overcome this hurdle, we turned to large genomic datasets to determine if there was convergent data correlating the loss of LDAH to any of the observed phenotypes. Analyses of both GWAS and eQTL indicate that a common variant rs13385191 at the LDAH locus is associated with a down-regulation of LDAH and a significant association with PCa in multiple genetic backgrounds. Further analysis of both prostate tissues and cell lines showed a positive correlation between tumorigenic tissues and LDAH downregulation. As a whole, this represents four independent lines of evidence from forward-genetic studies (i.e. rare de novo disruption of LDAH in DGAP056 with $\mathrm{PCa}$, significant association of a common variant at the LDAH locus and consistent eQTL in GWAS, down-regulation in prostate tumor tissues and downregulation in PCa cell lines) that all converge on an association between down-regulation of $L D A H$ and increased risk PCa, implicating a role of $L D A H$ in $\mathrm{PCa}$ tumor suppression.

To validate this hypothesis, we investigated the consequences of disruption of LDAH in two independent reversegenetic experiments. First, we investigated changes to $L D A H$ in vitro, which showed that reduced expression in benign human prostate cell lines showed increased rates of proliferation, while increasing expression in tumorigenic cell lines showed protection against both proliferation and migration. Second, we investigated the effect of Ldah knockout in vivo, which showed increased rate of $\mathrm{mPIN}$, an early precursor to $\mathrm{PCa}$, 
in the mouse prostate. While only a moderate increase over background (OR > 3.0), the $\mathrm{Ldah}^{-/}$mouse model was remarkable in that there were several observations of more severe invasive lesions, a rare observation for any tumor suppressor model and only observed in one other series of monogenic knockout mice models (Supplementary Material, Table S2) (28). These observations suggest that LDAH has similar properties to an early initiation tumor suppressor and could be explanative of DGAP056's early-onset PCa.

In addition to an increase in prostate lesions, our $\mathrm{Ldah}^{-/}$ mouse model developed SNHL and increased body mass. The observed auditory defect was more dramatic in the $\mathrm{Ldah}^{-/}$males and overlapped with the phenotype first observed in DGAP056, although hearing loss in male $\mathrm{Ldah}^{-/}$mice was less severe (10-30 dB loss versus $>90 \mathrm{~dB}$ ) and had a later onset (1 year versus congenital) than DGAP056. The increase in $\mathrm{Ldah}^{-1}$ body mass overlaps with a previous human GWAS, which found a nominal association between SNP rs4971547, located between exons 4 and 5 of LDAH and increased brachial arm circumference in females (29). Interestingly, there appeared to be a sex dependence in both traits, as SNHL was more dramatic in males and increased body mass was only significant in females. Contrary to our observations, Kory et al. (30) also report the generation of an $\mathrm{Ldah}^{-/}$mouse model, but fail to report any significant observations, which may be attributed to the fact that none of the phenotypes measured in this study (i.e. PCa, SNHL and body weight) were interrogated to the same degree. Further interrogation of these in vitro and in vivo models will be important for deciphering the functional consequence of changes in LDAH especially given that the function of LDAH is still unknown.

While it has been shown that LDAH co-localizes with lipid droplets, the function of this protein is currently debated (31). Five studies have now investigated the molecular function of LDAH with inconsistent findings $(30,32-35)$. Goo et al. $(33,35)$ found that in both macrophages and human embryonic kidney (HEK) cells, LDAH acts as a cholesteryl esterase at the surface of lipid droplets. However, Thiel et al. (32), Kory et al. (30) and Kolkhof et al. (34) contest the possibility of LDAH acts as a cholesteryl esterase. While Kory et al. (30) were unable to identify any functional consequences due to loss of LDAH, Theil et al. (32) and Kolkhof et al. (34) observe changes to lipid droplet architecture without any discernable lipid metabolism change, leading them to postulate that LDAH likely acts more as an acyltransferase, modifying phospholipids or an interaction hub in lipid droplets. In our studies, we found no evidence to either support or contradict the metabolic function of LDAH, but the localization to lipid droplets and putative role in lipid metabolism is intriguing as prostate tumorigenesis, hearing loss and obesity are associated with lipid dysregulation (36-38). Establishing the function of LDAH should help gain insight into this lipid oncogenic mechanism.

Given the cumulative evidence, our interpretation is that loss of LDAH increases the risk of PCa and may have other pleiotropic effects including SNHL. Its pathogenicity likely operates through haploinsufficiency in a lipid metabolic pathway contributing to the abnormal phenotype in DGAP056. Although the mouse phenotype attributed to Ldah knockout shows a later onset hearing loss, mouse models are not uncommonly less resistant to haploinsufficiency than humans and show variable expression. Furthermore, the mouse prostate is extremely resistant to metastatic cancer. A polygenic etiology could also be at play given the genetic complexity of both PCa and SNHL, and it is possible that the genetic background of DGAP056 and/or other genes dysregulated by the translocation could impact the phenotype.
Of note in this regard, targeted exome sequencing of hearing loss genes was negative for pathogenic variants, although such tests are limited by our knowledge of an incomplete catalog of genes for hearing. Nonetheless, whether the complex DGAP056 phenotype is the result of simple haploinsufficiency or polygenic effects, the abundance of evidence argues in support of a loss of LDAH expression as a risk for both PCa and SNHL.

In summary, we report that loss of LDAH results in higher risk of developing PCa, which was first identified using a convergent genomics approach and then verified both in vitro and in vivo models. We propose that the $\mathrm{Ldah}^{-/}$mouse model will facilitate further investigations into the role of LDAH in lipid biology and help define the molecular mechanism involved in tumor initiation and metastasis during prostate oncogenesis. The LDAH pathway may be a new potential target for treatment of PCa.

\section{Materials and Methods}

\section{Human subject}

DGAP056 was enrolled in the DGAP under the Partners HealthCare System Internal Review Board (IRB) protocol number 1999P003090. Karyotyping was performed on leukocytes collected from DGAP056, using GTG-banding per standard protocols. An LCL was generated from DGAP056 peripheral blood as a source for all additional DNA, RNA and protein assays. FISH was performed on metaphase chromosomes from at least 10 cells prepared from DGAP056 and control LCLs using BACs directly labeled with either SpectrumOrange- or SpectrumGreen-conjugated nucleotides by nick translation, and differently labeled pairs were hybridized to metaphase chromosomes per standard protocols and analyzed with Cytovision (39). Translocation breakpoints were further refined by restriction endonuclease cleavage mapping and targeted with radioactively labeled probes on southern blots. Putative DGAP056 breakpoints were screened using a suppression PCR strategy and confirmed with Sanger sequencing of the region to achieve breakpoint resolution (40). Chromosomal breakpoints are described using Next-Generation Cytogenetic Nomenclature obtained by BLA(S)T Output Sequence Tool of Nomenclature (7). Unique RNA transcripts were analyzed by performing the 3'-RACE reactions and gel-purified products were Sanger sequenced.

\section{Custom-made anti-LDAH antibody, tissue microarrays and immunohistochemistry}

A custom rabbit polyclonal anti-Ldah antibody was developed with Covance (Dedham, MA) based on predicted antigenicity of three polypeptide immunogens ([H]-SVTPKDKKVLAAPQEESNA[NH $\mathrm{N}_{2}$ ], [H]-GQIEHKIAFLRAHVPKDVKL-[ $\left[\mathrm{NH}_{2}\right]$ and [H]-PVKYYEDM KKDFPEG-[NH $\mathrm{NH}_{2}$ ) against 1110057K04Rik (MGI:1916082, the mouse LDAH ortholog and referred to as Ldah herein) using the Covance 77 days protocol. Sensitivity and specificity of antiLDAH by western blots on both LDAH and Ldah transfected cells as well as from $\mathrm{Ldah}^{+/+}$and $\mathrm{Ldah}^{-/}$tissues (Supplementary Material, Fig. S6) were compared against expected full-length protein size $(\sim 38 \mathrm{kDa})$ and a commercial LDAH antibody (HPA034730, Human Protein Atlas). This custom anti-LDAH antibody was used to assess prostate tissue microarrays (TMAs) that were generously provided by Dr Massimo Loda containing 88 cores from 16 patients. Immunohistochemistry (IHC) was performed on the TMAs using a heat-induced epitope retrieval protocol with a 1:2000 dilution for anti-Ldah and 1:200 (per 
manufacturer recommendation) for the commercial antibody, labeled with biotinylated universal secondary antibody and visualized with 3,3'-diaminobenzidine chromogen staining. Analysis of TMAs was performed by two protocols to avoid analysis bias-manually, with visual scoring by a pathologist, and semi-automated, with computational image processing of regions-of-interest using ImageJ.

\section{Prostate cell lines}

Prostate cell lines were obtained from the American Type Culture Collection (ATCC, Manassas, VA) or generously provided by Dr Shaoyong Chen, and cultured according to ATCC-specified conditions. Overexpression of $L D A H$ was performed with a custom construct containing LDAH full-length cDNA on MSCV-PIG backbone (LDAH+) while knock-down of LDAH was performed with shRNA targeting LDAH. For cell proliferation assays, plated cells were fixed and stained with $0.1 \%$ crystal violet, followed by dye extraction and optical density measurement at $595 \mathrm{~nm}$ in triplicate for each experimental parameter. To determine migration and invasion, plated cells were detached and placed into the top chamber of $8 \mu \mathrm{m}$ transwell inserts (migration assay) or Matrigelcoated transwell inserts (invasion assay). After 24 or $48 \mathrm{~h}$, cells were counted in three microscopic fields in three independent assays.

\section{Mouse model generation}

$\mathrm{Ldah}^{-/}$mouse models were generated using a homologous recombination (HR) construct containing a FlipRosaßgeo cassette into intron 2 of the mouse LDAH ortholog, 1110057K04Rik, using Gateway Technology (Supplementary Material, Fig. S6) (23) embedded in constructs kindly donated by the Beier Laboratory (Division of Genetics, BWH, Boston, MA). Mouse embryonic stem cells (ESCs, strain 129S4/SvJae) were microinjected with the HR construct, PCR-screened, C57BL/6J blastocyst-injected and implanted into pseudopregnant females. Filial 0 (F0) generation were bred with B6 mice and the F1 generation screened for $\mathrm{Ldah}^{-}$allele. We subsequently backcrossed $\mathrm{Ldah}^{-/}$to CBA mice, creating odd-numbered generations of $\mathrm{Ldah}^{+/}$, and intercrossed $\mathrm{Ldah}^{+/-}$littermates, creating even-numbered generations of all three genotypes, for multiple generations. Gross necropsies and tissue harvest were performed on 1-year-old mice.

\section{Mouse physiology and histology}

Mice were evaluated for differences in body weight, cochlear function and prostate histology based on initial gross observations and/or suspected phenotypes based on human subject studies. $\mathrm{Ldah}^{-/}$and $\mathrm{Ldah}^{+/+}$littermate mice were weighed on a digital scale the first week of every month to determine body mass, and weight-over-time was analyzed for each mouse, sex and genotype, trimmed for outliers using the Robust Regression and Outlier algorithm, and compared using the extra sum-ofsquares F-test (41). Mouse auditory evaluations were performed using both distortion-product otoacoustic emissions (DPOAEs) and ABRs in $\mathrm{Ldah}^{-/}$and in $\mathrm{Ldah}^{+/+}$littermates to assess cochlear and auditory neural function. Data from ears were only used in final analyses if both ABRs and DPOAEs were performed and cochlea was normal on visual and histological inspection. We assessed statistical significance with two-way ANOVA (genotype, frequency), and as no interaction effects were significant, only the main effect of interest (i.e. genotype) is reported. For histology, prostate tissues were microdissected to isolate AP, VP and DLP lobes, placed in labeled cassettes and fixed in $10 \%$ formalin for at least $24 \mathrm{~h}$, followed by paraffin-embedding, sectioning and $\mathrm{H} \& \mathrm{E}$-staining of a subset. Two independent pathologists examined H\&E- and Ki-67-stained prostate lobes for each mouse and graded them using a system based on Shappell et al. (25) and Park et al. (26) to score the lobe as either prostatic intraepithelial neoplasia (mPIN, with sub-grading to MPIN I-IV), microinvasive or invasive lesion(s) (25). The most advanced lesion for each lobe was used in the statistical calculations.

\section{Western blots and qPCR}

For western blots, protein was extracted by suspending tissues or cells in cold radioimmunoprecipitation assay buffer and protease inhibitor, homogenized and centrifuged (15 $000 \mathrm{~g}$ for $30 \mathrm{~min}$ at $4^{\circ} \mathrm{C}$ ). Equal amounts of protein (as assessed by Quick Start ${ }^{\mathrm{TM}}$ Bradford assay system, Bio-Rad, Hercules, CA) were used for western blots performed in $4-12 \%$ Tris-Bis gel, transferred to polyvinylidene difluoride membrane and probed using custom anti-Ldah antibody with secondary horseradish peroxidasetagged antibody and imaged with chemiluminescence for at least three biological replicates of each tissue. RNA was prepared following suspension of tissues or cells in cold TRIzol ${ }^{\circledR}$, homogenized and TRIzol ${ }^{\circledR}$ extracted coupled with secondary RNeasy kit with On-Column DNAse digestion per manufacturer protocol. Quantitative real-time PCR ( $q$ RT-PCR) was performed using RNA isolated from at least three biological replicates converted to cDNA with either qScript cDNA SuperMix or SuperScriptIII. The PCR reaction was performed with Perfecta SYBR-Green SuperMix for iQ on an iCycler IQ or CFX Connect ${ }^{\text {TM }}$ Real-Time PCR Detection System (Bio-Rad, Hercules, CA) and calculated with relative gene expression using the $\Delta \Delta$ CT method.

\section{GWAS, eQTL, microarray and PRT analysis}

Data for meta-analysis of genetic studies were curated by searching PubMed using the following terms: LDAH, C2orf43, FLJ21820, UPF0554 and 1110057K04Rik (last accessed June 2017). Search results were perused to identify association studies with any genetic markers. Meta-analysis was performed using a fixed effects inverse variance model. For eQTL data were acquired for 87 prostate samples from GTEx (www.gtexportal.org) and eQTL analysis performed using the MatrixEQTL R package (42). The SNP rs13385191 was computed against LDAH transcripts ENST00000237822.3, ENST00000381090.3, ENST00000470099.1, ENST00000419825.2, ENST00000402479.2, ENST00000403006.2, ENST00000412261.1, ENST00000432947.1, ENST00000435420.2, ENST00000440866.2, ENST00000541941.1, while correcting for age, race and ethnicity. For meta-analysis of PCa expression arrays, data were curated from GEO by filtering for large prostate datasets that compared non-laser captured microarray expression analysis. A custom R script was used to adjust for skew in reported results (using $\log _{2}$ correction where needed) and quantile normalized $(43,44)$. LDAH quantile normalized values were used to calculate $z$-values within each study, for cross-platform comparisons and to rank order differential expression across the entire genome. For the PRT, the gene plexus uses a set of genomic elements to define regions that affect the gene's function (17). In brief, the normal prostate plexus for LDAH is generated by defining a unique set of tiles (based on chromatin state, Hi-C data, transcription start site and exons) based on a local expectation of interaction frequency representing all of the proximal and distal elements that 
impinge on a gene's function. This plexus was used to retrieve tumor mutations from 55 whole genome tumor-normal pairs for prostate adenocarcinoma from the GTEx repository. The PRT permutation-based algorithm estimates the expected number of mutations for a gene's plexus and computes an enrichment score and $P$-value for statistical significance of positive selection. We performed three independent tests in the LDAH plexus for promoter, dyadic and enhancer classes of regulatory elements, with P-values of $0.004,0.023$ and 0.492 , respectively.

\section{Supplementary Material}

Supplementary Material is available at HMG online.

\section{Acknowledgements}

We thank DGAP056 and his family for their participation in this study. We would also like to thank M. Loda, C. Abate-Shen and R. Kucherlapati for their guidance in understanding the PCa phenotype, R. Robertson for his analysis of the MRI, S. Chen for providing the C4-2 prostate cell line and B. Bjork along with D. Beier for providing the pFlipRosa $\beta$ geo-ENTR11 and pPNRDEST-R4-R3 constructs for engineering the mouse model. Several core facilities were used in this study: DF/HCC DNA Resource Core, DF/HCC Rodent Histopathology Core, Biopolymers Facility at Harvard Medical School, BWH CytoGenomics Core and BWH Transgenic Core.

Conflict of Interest statement. The authors report no conflict of interest with the production of this manuscript.

\section{Funding}

R01 DC003402 (to C.C.M.), P01 GM061354 (to C.C.M.), T32 GM007748 (to B.B.C. and C.C.M.), F32 DC012466 (to B.B.C.), F31 DC007540 (to K.K.B.), F31 DC005712 (to R.E.W.), P30 DC005209 (to M.C.L.), R01 DC00188 (to M.C.L.), K01 DK093638 (to A.S.B.), Giovanni Armenise-Harvard Foundation Career Development Award (to A.L.) and P50 CA092629 (to M.C., A.L., M.R. and P.P.P.) and C.C.M. is supported by the NIHR Manchester Biomedical Research Centre.

\section{References}

1. Fatkin, D., Seidman, C.E. and Seidman, J.G. (2014) Genetics and disease of ventricular muscle. Cold Spring Harb. Perspect. Med., 4, a021063.

2. Manolio, T.A., Collins, F.S., Cox, N.J., Goldstein, D.B., Hindorff, L.A., Hunter, D.J., McCarthy, M.I., Ramos, E.M., Cardon, L.R., Chakravarti, A. et al. (2009) Finding the missing heritability of complex diseases. Nature, 461, 747-753.

3. Maher, B. (2008) Personal genomes: the case of the missing heritability. Nature, $456,18-21$.

4. Claussnitzer, M., Dankel, S.N., Kim, K.H., Quon, G., Meuleman, W., Haugen, C., Glunk, V., Sousa, I.S., Beaudry, J.L., Puviindran, V. et al. (2015) FTO obesity variant circuitry and adipocyte browning in humans. N. Engl. J. Med., 373, 895-907.

5. Talkowski, M.E., Rosenfeld, J.A., Blumenthal, I., Pillalamarri, V., Chiang, C., Heilbut, A., Ernst, C., Hanscom, C., Rossin, E., Lindgren, A.M. et al. (2012) Sequencing chromosomal abnormalities reveals neurodevelopmental loci that confer risk across diagnostic boundaries. Cell, 149, 525-537.

6. Ayalew, M., Le-Niculescu, H., Levey, D.F., Jain, N., Changala, B., Patel, S.D., Winiger, E., Breier, A., Shekhar, A., Amdur, R. et al. (2012) Convergent functional genomics of schizophrenia: from comprehensive understanding to genetic risk prediction. Mol. Psychiatry, 17, 887-905.

7. Ordulu, Z., Wong, K.E., Currall, B.B., Ivanov, A.R., Pereira, S., Althari, S., Gusella, J.F., Talkowski, M.E. and Morton, C.C. (2014) Describing sequencing results of structural chromosome rearrangements with a suggested nextgeneration cytogenetic nomenclature. Am. J. Hum. Genet., 94, 695-709.

8. Li, M.J., Liu, Z., Wang, P., Wong, M.P., Nelson, M.R., Kocher, J.P., Yeager, M., Sham, P.C., Chanock, S.J., Xia, Z. et al. (2016) GWASdb v2: an update database for human genetic variants identified by genome-wide association studies. Nucleic Acids Res., 44, D869-D876.

9. Welter, D., MacArthur, J., Morales, J., Burdett, T., Hall, P., Junkins, H., Klemm, A., Flicek, P., Manolio, T., Hindorff, L. et al. (2014) The NHGRI GWAS catalog, a curated resource of SNPtrait associations. Nucleic Acids Res., 42, D1001-D1006.

10. Takata, R., Akamatsu, S., Kubo, M., Takahashi, A., Hosono, N., Kawaguchi, T., Tsunoda, T., Inazawa, J., Kamatani, N., Ogawa, O. et al. (2010) Genome-wide association study identifies five new susceptibility loci for prostate cancer in the Japanese population. Nat. Genet., 42, 751-754.

11. Long, Q.Z., Du, Y.F., Ding, X.Y., Li, X., Song, W.B., Yang, Y., Zhang, P., Zhou, J.P. and Liu, X.G. (2012) Replication and fine mapping for association of the C2orf43, FOXP4, GPRC6A and RFX6 genes with prostate cancer in the Chinese population. PLoS One, 7, e37866.

12. Haiman, C.A., Chen, G.K., Blot, W.J., Strom, S.S., Berndt, S.I., Kittles, R.A., Rybicki, B.A., Isaacs, W.B., Ingles, S.A., Stanford, J.L. et al. (2011) Characterizing genetic risk at known prostate cancer susceptibility loci in African Americans. PLoS Genet., 7, e1001387.

13. Lindstrom, S., Schumacher, F.R., Campa, D., Albanes, D., Andriole, G., Berndt, S.I., Bueno-de-Mesquita, H.B., Chanock, S.J., Diver, W.R., Ganziano, J.M. et al. (2012) Replication of five prostate cancer loci identified in an Asian populationresults from the NCI Breast and Prostate Cancer Cohort Consortium (BPC3). Cancer Epidemiol. Biomarkers Prev., 21, 212-216.

14. Wang, N.N., Xu, Y., Yang, K., Wei, D., Zhang, Y.G., Liu, M., Shi, X.H., Liang, S.Y., Sun, L., Zhu, X.Q. et al. (2013) Susceptibility loci associations with prostate cancer risk in northern Chinese men. Asian Pac. J. Cancer Prev., 14, 3075-3078.

15. Shui, I.M., Lindstrom, S., Kibel, A.S., Berndt, S.I., Campa, D., Gerke, T., Penney, K.L., Albanes, D., Berg, C., Bueno-deMesquita, H.B. et al. (2014) Prostate cancer (PCa) risk variants and risk of fatal PCa in the National Cancer Institute Breast and Prostate Cancer Cohort Consortium. Eur. Urol., 65, 1069-1075.

16. Penney, K.L., Pyne, S., Schumacher, F.R., Sinnott, J.A., Mucci, L.A., Kraft, P.L., Ma, J., Oh, W.K., Kurth, T., Kantoff, P.W. et al. (2010) Genome-wide association study of prostate cancer mortality. Cancer Epidemiol. Biomarkers Preu., 19, 2869-2876.

17. Sallari, R.C., Sinnott-Armstrong, N.A., French, J.D., Kron, K.J., Ho, J., Moore, J.H., Stambolic, V., Edwards, S.L., Lupien, M. and Kellis, M. (2016) Convergence of dispersed regulatory mutations predicts driver genes in prostate cancer. bioRxiv, 10.1101/097451.

18. Penney, K.L., Sinnott, J.A., Tyekucheva, S., Gerke, T., Shui, I.M., Kraft, P., Sesso, H.D., Freedman, M.L., Loda, M., Mucci, L.A. et al. (2015) Association of prostate cancer risk variants with gene expression in normal and tumor tissue. Cancer Epidemiol. Biomarkers Prev., 24, 255-260. 
19. Lapointe, J., Li, C., Higgins, J.P., van de Rijn, M., Bair, E., Montgomery, K., Ferrari, M., Egevad, L., Rayford, W., Bergerheim, U. et al. (2004) Gene expression profiling identifies clinically relevant subtypes of prostate cancer. Proc. Natl. Acad. Sci. U. S. A., 101, 811-816.

20. Taylor, B.S., Schultz, N., Hieronymus, H., Gopalan, A., Xiao, Y., Carver, B.S., Arora, V.K., Kaushik, P., Cerami, E., Reva, B. et al. (2010) Integrative genomic profiling of human prostate cancer. Cancer Cell, 18, 11-22.

21. Grasso, C.S., Wu, Y.M., Robinson, D.R., Cao, X., Dhanasekaran, S.M., Khan, A.P., Quist, M.J., Jing, X., Lonigro, R.J., Brenner, J.C. et al. (2012) The mutational landscape of lethal castrationresistant prostate cancer. Nature, 487, 239-243.

22. De Marzo, A.M., Putzi, M.J. and Nelson, W.G. (2001) New concepts in the pathology of prostatic epithelial carcinogenesis. Urology, 57, 103-114.

23. Schnutgen, F., De-Zolt, S., Van Sloun, P., Hollatz, M., Floss, T., Hansen, J., Altschmied, J., Seisenberger, C., Ghyselinck, N.B., Ruiz, P. et al. (2005) Genomewide production of multipurpose alleles for the functional analysis of the mouse genome. Proc. Natl. Acad. Sci. U. S. A., 102, 7221-7226.

24. Ittmann, M., Huang, J., Radaelli, E., Martin, P., Signoretti, S., Sullivan, R., Simons, B.W., Ward, J.M., Robinson, B.D., Chu, G.C. et al. (2013) Animal models of human prostate cancer: the consensus report of the New York meeting of the Mouse Models of Human Cancers Consortium Prostate Pathology Committee. Cancer Res., 73, 2718-2736.

25. Shappell, S.B., Thomas, G.V., Roberts, R.L., Herbert, R., Ittmann, M.M., Rubin, M.A., Humphrey, P.A., Sundberg, J.P., Rozengurt, N., Barrios, R. et al. (2004) Prostate pathology of genetically engineered mice: definitions and classification. The consensus report from the bar harbor meeting of the Mouse Models of Human Cancer Consortium Prostate Pathology Committee. Cancer Res., 64, 2270-2305.

26. Park, J.H., Walls, J.E., Galvez, J.J., Kim, M., Abate-Shen, C., Shen, M.M. and Cardiff, R.D. (2002) Prostatic intraepithelial neoplasia in genetically engineered mice. Am. J. Pathol., 161, 727-735.

27. Berquin, I.M., Min, Y., Wu, R., Wu, H. and Chen, Y.Q. (2005) Expression signature of the mouse prostate. J. Biol. Chem., 280, 36442-36451.

28. Wang, S., Gao, J., Lei, Q., Rozengurt, N., Pritchard, C., Jiao, J., Thomas, G.V., Li, G., Roy-Burman, P., Nelson, P.S. et al. (2003) Prostate-specific deletion of the murine Pten tumor suppressor gene leads to metastatic prostate cancer. Cancer Cell, 4, 209-221.

29. Boraska, V., Day-Williams, A., Franklin, C.S., Elliott, K.S., Panoutsopoulou, K., Tachmazidou, I., Albrecht, E., Bandinelli, S., Beilin, L.J., Bochud, M. et al. (2012) Genome-wide association study to identify common variants associated with brachial circumference: a meta-analysis of 14 cohorts. PLoS One, 7, e31369.

30. Kory, N., Grond, S., Kamat, S.S., Li, Z., Krahmer, N., Chitraju, C., Zhou, P., Frohlich, F., Semova, I., Ejsing, C. et al. (2017) Mice lacking lipid droplet-associated hydrolase, a gene linked to human prostate cancer, have normal cholesterol ester metabolism. J. Lipid Res., 58, 226-235.

31. Wan, H.C., Melo, R.C., Jin, Z., Dvorak, A.M. and Weller, P.F. (2007) Roles and origins of leukocyte lipid bodies: proteomic and ultrastructural studies. FASEB J., 21, 167-178.

32. Thiel, K., Heier, C., Haberl, V., Thul, P.J., Oberer, M., Lass, A., Jackle, H. and Beller, M. (2013) The evolutionarily conserved protein CG9186 is associated with lipid droplets, required for their positioning and for fat storage. J. Cell. Sci., 126, 2198-2212.

33. Goo, Y.H., Son, S.H., Kreienberg, P.B. and Paul, A. (2014) Novel lipid droplet-associated serine hydrolase regulates macrophage cholesterol mobilization. Arterioscler. Thromb. Vasc. Biol., 34, 386-396.

34. Kolkhof, P., Werthebach, M., van de Venn, A., Poschmann, G., Chen, L., Welte, M., Stuhler, K. and Beller, M. (2017) A Luciferase-fragment complementation assay to detect lipid droplet-associated protein-protein interactions. Mol. Cell Proteomics, 16, 329-345.

35. Goo, Y.H., Son, S.H. and Paul, A. (2017) Lipid dropletassociated hydrolase promotes lipid droplet fusion and enhances ATGL degradation and triglyceride accumulation. Sci. Rep., 7, 2743.

36. Zadra, G., Photopoulos, C. and Loda, M. (2013) The fat side of prostate cancer. Biochim. Biophys. Acta., 1831, 1518-1532.

37. Urrutia, R.A. and Kalinec, F. (2015) Biology and pathobiology of lipid droplets and their potential role in the protection of the organ of Corti. Hear Res., 330, 26-38.

38. Krahmer, N., Farese, R.V. Jr. and Walther, T.C. (2013) Balancing the fat: lipid droplets and human disease. EMBO Mol. Med., 5, 973-983.

39. Ney, P.A., Andrews, N.C., Jane, S.M., Safer, B., Purucker, M.E., Weremowicz, S., Morton, C.C., Goff, S.C., Orkin, S.H. and Nienhuis, A.W. (1993) Purification of the human NF-E2 complex: cDNA cloning of the hematopoietic cell-specific subunit and evidence for an associated partner. Mol. Cell Biol., 13, 5604-5612.

40. Siebert, P.D., Chenchik, A., Kellogg, D.E., Lukyanov, K.A. and Lukyanov, S.A. (1995) An improved PCR method for walking in uncloned genomic DNA. Nucleic Acids Res., 23, 1087-1088.

41. Motulsky, H.J. and Brown, R.E. (2006) Detecting outliers when fitting data with nonlinear regression - a new method based on robust nonlinear regression and the false discovery rate. BMC Bioinformatics, 7, 123.

42. Shabalin, A.A. (2012) Matrix eQTL: ultra fast eQTL analysis via large matrix operations. Bioinformatics, 28, 1353-1358.

43. Barrett, T., Wilhite, S.E., Ledoux, P., Evangelista, C., Kim, I.F., Tomashevsky, M., Marshall, K.A., Phillippy, K.H., Sherman, P.M., Holko, M. et al. (2013) NCBI GEO: archive for functional genomics data sets-update. Nucleic Acids Res., 41, D991-D995.

44. Bolstad, B.M., Irizarry, R.A., Astrand, M. and Speed, T.P. (2003) A comparison of normalization methods for high density oligonucleotide array data based on variance and bias. Bioinformatics, 19, 185-193. 